This voltage is amplified and impressed on a cathode ray oscillograph, which has in connexion with it a properly synchronised time-axis device. The hammer is driven by a synchronous motor and strikes the end of the rod ten times a second. By proper adjustment throughout, each train of waves occurs on the oscillograph screen exactly superposed on the preceding train, so that a steady picture is obtained representative of the periodic displacements of the end of the rod with time. This graph, as it really is, may be photographed if desired.

It has been found that in most cases the waves are quite. complex, being combinations of longitudinal, flexural, and possibly other modes of vibration. In order to separate these component frequencies, the voltage from the amplifier may be passed through a tuned circuit and the selected frequency then impressed on the oscillograph. It is thus possible to pick out vibrations of different types.

In Fig. 1 are shown several examples of waves which occur in duralumin rods when they are struck with a hammer. The fourth curve shows the predominating frequency present in the wave immediately above it, this analysis being obtained with the use of the tuned circuit previously mentioned.

By measuring the decrement of any desired wave, the coefficient of solid viscosity corresponding to that frequency may be calculated, and it is believed that considerably greater accuracy may be obtained in this way than has been possible with previous, more indirect methods. From the fourth curve in Fig. 1, which corresponds to the fundamental mode of longitudinal vibration for a certain duralumin rod, the logarithmic decrement was found to be 0.016 , giving a value for the coefficient of solid viscosity of $4.6 \times 10^{4}$. Although this rod was of duralumin, it is interesting to note that in $1922 \mathrm{Dr}$. R. W. Boyle ${ }^{1}$ suggested that when the coefficient was accurately determined for steel (in many respects similar to duralumin), its order would probably be not greater than $10^{5}$. The variation of this coefficient with frequency and amplitude of vibration remains to be determined, but when this matter is examined in greater detail, it is probable that a clearer understanding of the nature of solid viscosity, including hysteresis, may be forthcoming.

GeORge S. Fired.

National Research Laboratories,

Ottawa, June 8.

Trans. Roy. Soc. Can., Sect. III., p. 293 ; 1932.

\section{Mean Lives of the Lowest Metastable States in Neutral Oxygen, and Intensities of Lines Arising therefrom}

Transitions from the lowest metastable levels in the Or spectrum are of interest as occurring in nebular and auroral spectra. The red lines ${ }^{3} P_{2,1}-{ }^{1} D_{2}$ $(\lambda \lambda .6300,6364)$, which have been produced in the laboratory by Hopfield, 1 have been identified by Paschen ${ }^{2}$ as occurring in the spectra of some of the nebulæ, and by Sommer ${ }^{3}$ as occurring in the spectrum of the night sky, while the line ${ }^{1} D_{2}-{ }^{1} S_{0}(\lambda 5577)$ is the well-known auroral green line first identified by McLennan. A theoretical calculation of the intensities of such transitions and the mean lives of the metastable states is therefore of interest.

These 'forbidden' transitions are evidently due to quadripole radiation, for the Zeeman effect in the auroral green line has been shown by Frerichs and Campbell ${ }^{4}$ to be in agreement with the supposition of quadripole radiation, but inconsistent with the hypothesis of perturbed dipole radiation. In a forthcoming paper in the Proceedings of the Royal Society, I have made the corresponding calculations for OIII with reference to nebular lines, on the assumption of quadripole radiation. ${ }^{5}$ The final results are expressed in terms of integrals of radial wave functions, thus depending only on the central field of force adopted, and these integrals can be calculated from a comparison of calculated and observed separations between the levels. Now, Heisenberg ${ }^{6}$ has shown recently that the wave equation for the lowest levels of an atom in which there are $n$ electrons outside closed shells only differs, in first approximation, from that of an atom in which there are $n$ 'holes' (that is, a closed shell is incomplete by $n$ electrons) in that a different central field of force must be adopted. This enables the method outlined above for OIII to be applied immediately to the case in hand, thus yielding the following results for the $2 p^{4}$ terms in Or :

$$
\begin{gathered}
\text { Line. } \\
{ }^{3} P_{2}-{ }^{1} D_{2}(\lambda 6300) \\
{ }^{3} P_{1}-{ }^{1} D_{2}(\lambda 6364) \\
{ }^{3} P_{0}-{ }^{1} D_{2}(\lambda 6391) \\
{ }^{1} D_{2}-{ }^{1} S_{0}(\lambda 5577) \\
{ }^{3} P_{2}-{ }^{1} S_{0} \text { (not observed) } \\
{ }^{3} P_{1}-{ }^{1} S_{0} \quad, \\
{ }^{3} P_{0}-{ }^{1} S_{0} \quad ",
\end{gathered}
$$

Relative Intensity (per atom)

Relative intensity of ${ }^{1} D_{2}-{ }^{1} S_{0}$ to ${ }^{8} \mathrm{P}_{2}-{ }^{1} D_{2}=1400: 1$

Mean life of ${ }^{1} S_{0}$ state $=0.02 \mathrm{sec}$.

The results are, of course, only approximate, the figures for ${ }^{1} D_{2}-{ }^{1} S_{0}$ and the mean life of the ${ }^{1} S_{0}$ state being less accurate than the others. The only direct comparison with observation is in the relative intensity of lines with ${ }^{1} D_{2}$ as initial state. The relative intensity of ${ }^{3} P_{2}-{ }^{1} D_{2}$ is about $4: 1$ according to Hopfield, ${ }^{1}$ while for the nebula N.G.C. 7027 the ratio is about $5: 2$ according to Wright. ${ }^{2}$ The line ${ }^{3} P_{0}-{ }^{1} D_{2}$ ( $\lambda 6391)$ is not observed in the nebulæ, but was weakly observed in the laboratory according to Paschen. These results are in satisfactory agreement with the above. In a subsequent paper, however, Paschen ${ }^{7}$ gives the relative intensity of ${ }^{3} P_{2}{ }{ }^{-1} D_{2}$ as $5: 3: 1$. If this were really the case, it would, of course, be in serious disagreement with the theory.

The figures for the mean lives throw some light on conditions in the nebulæ and the auroral regions. If it becomes definitely established that the red lines $(\lambda \lambda 6300,6364)$ occur in the auroral spectrum, the observed relative intensity of $\lambda 5577$ to $\lambda 6300$, combined with the above calculated relative intensity per atom, would furnish the relative number of atoms in the ${ }^{1} S_{0}$ and ${ }^{1} D_{2}$ states, which would be of interest in connexion with possible processes of excitation in the upper atmosphere.

I wish to thank Mr. M. F. Crawford for valuable discussion of the experimental data.

Department of Applied Mathematics, A. F. Stevenson. University of Toronto, May 26.

1 Hopfield, Phys. Rev., 37, $100 ; 1931$.

- Paschen, Naturwiss., 18, 752; 1930.

3 Sommer, ibid. Cf. Grotrian, Naturwiss., 20, 85 ; 1932.

- Frerichs and Campbell, Phys. Rev., 36, 1460; 1931.

5 Of. also Bartlett, Phys. Rev., 34, 1247; 1929.

- Heisenberg, Ann. Phys., 10, 888; 1931 .

1 Paschen, Z. Physik, 65, 1; 1930.

\section{Layer-chain Structures of Thallium Di-Alkyl Halides}

During the last year we have been studying the crystal structures of a series of compounds $R_{2}$ TIX, which prove to be of some interest in view of the recent work on the rotation of molecules in crystals. The dimethyl thallium halides are tetragonal and the others orthorhombic, pseudo-tetragonal, and all show 